\title{
Impulse Response Analysis in a Low Voltage Circuit Breaker using Mathematical Modelling
}

\author{
Senthil Kumar Mouleeswaran', Suryanarayanan Murali ${ }^{2 *}$, M. Bagyalakshmi ${ }^{3}$, \\ V. Pradeep Kumar ${ }^{4}$
}

\author{
${ }^{1}$ Department of Mechanical Engineering, PSG College of Technology, Coimbatore, India \\ ${ }^{2}$ Department of Mechanical Engineering, PSG College of Technology, Coimbatore, India \\ ${ }^{3}$ Department of Applied Mathematics and Computation Sciences, PSG College of Technology, Coimbatore, India \\ ${ }^{4}$ Switchgear Design and Development Centre, Larsen \& Toubro Limited, Malumichampatty campus, Coimbatore, India
}

\section{BIOGRAPHICAL NOTES}

Senthil kumar Mouleeswaran born in the year 1973, is currently working as Assistant Professor in the Department of Mechanical Engineering at PSG College of Technology, India. He has about 16 years of teaching experience and 1 year of industrial experience. He obtained his B.E. Degree in Mechanical Engineering (1994), M.E. Degree in Engineering Design (1996) and Ph.D. in Active Suspension System (2008). He has received ISTE National Award for "Best Research in the field of Machine design and Vibration by Young Teacher" in 2006. He has also received AICTE Career Award for his research on Particle Damping in 2009. He was awarded with Dr.Sundaramoorthy Award for Outstanding Academic contribution in 2011 from PSG College of Technology. He has authored about 50 journal papers and about 50 conference papers. He has successfully completed many sponsored research projects. He is active member of many professional bodies like ISTE, IE, TSI, etc. He has been actively involved in many consultancy works in the areas of design and vibration control. His fields of interest include vibration control, composites, smart structures, etc.

Suryanarayanan Murali born in the year 1992, is currently doing his B.E Mechanical Engineering in PSG College of Technology, Coimbatore, Tamil Nadu, India. The author is the Secretary of the Association of Mechanical Engineering in PSG College of Technology. He is active member of many professional bodies like ISTE, IE, ASME, etc. He has conducted training programme on Design of automation systems for Ashok Leyland Graduate Engineering Trainees. He has conducted many seminars for students on Pneumatic circuit design, Sensor interfacing using LabVIEW, etc. He has presented a paper titled "Determination of ergonomic positions for the design of therapeutic setup using virtual instrumentation and sensors" in the XXVIII National Convention of Mechanical Engineers conducted by the Institution of Engineers (India), Coimbatore Local Center held at PSG College Technology. He had done internship in LARSEN \& TOUBRO Ltd., under the Switchgear Design and Development centre and did a project on Impulse response analysis for nuisance tripping prevention which is being presented in this paper.

Ms.M.Bagyalakshmi was born in the year 1980. She completed her under graduation course B.Sc. Mathematics at Providence College for Women, Coonoor in 2000. She has completed her post graduation M.Sc. Mathematics as a Gold medalist at Bharathiyar University, Coimbatore in 2002. She did her M.Phil in the year 2003 under Madurai Kamaraj University. She started her teaching career as a lecturer at PSG college of Technology in 
the year 2006. She has seven years of teaching experience and has guided four UG projects. She has attended 10 National / International conferences and workshops in the area of Fluid Mechanics. She is currently working as an Assistant Professor in the Department of Applied Mathematics and Computational Sciences and pursuing research in the field of Fluid Mechanics.

Mr. Pradeepkumar Veluswamy born in the year 1986, is currently working as Assistant Manager in Switchgear Design \& Development Centre, L \& $T$ limited, India. He has about 5 years of industrial experience. He obtained his B.E. Degree in Mechanical Engineering (2008) at Park College of Engineering and Technology. He has been involved in new product development activities and also has experience in structural analysis of products.

\section{KEY WORDS}

Vibration prediction; Impact and shock analysis; Mathematical modeling; Low voltage circuit breaker; Nuisance tripping.

\section{ABSTRACT}

Circuit breakers are of inevitable use in this modern era of electronic and electrical boom. In this paper, the problem of "Nuisance tripping" caused due to environmental impact forces in circuit breakers is analyzed. Formerly, impact response of a circuit breaker latching system was analyzed only using through practical experimentation which proves to be very costly, given the cost of sophisticated equipment used for the testing and the associated employees' over head costs along with other indirect costs. In this research paper, a theoretical model was developed to determine the impact response of a circuit breaker system under the application of prescribed impact forces. This model can be used by a design engineer to predict the impact response of the designed circuit breaker latching setup. So, even before the manufacturing of trial runs begins, an engineer can be very sure that his component designed will withstand the standard impact forces. Hence, the above said costs incurred due to impact testing and the associated reworking costs can be neglected, or at least minimized to a considerable level. This research mainly focuses only on the methodology to be used with circuit breakers to predict and analyze the impact responses.

\section{Introduction}

Tripping is the process of disconnecting the device or load and the power source, at the circuit breaker. As the name suggests, nuisance tripping is something that happens in circuit breakers, wherein tripping occurs without the advent of the user tripping it. Or, in other words it is called so when the circuit breaker latches trips unnecessarily or without the need, causing the power in the line to trip when the loading device needs it. Since the components used in a circuit breaker are mostly mechanical or thermo-mechanical equipments, they are susceptible to mimic the forces that arise from the environment. The forces from the environment, either naturally induced (for example, earthquake, tsunami, etc) or induced by human work and processes (for example, industrial scenario) cause the components in the circuit breaker to displace in accordance to the applied forces being manipulated by some components such as springs, dampers, etc in the circuit breaker. These environmental forces may also cause the circuit breaker components to malfunction or to get damaged. This is a major problem because the circuit breaker should have the capacity to trip only when the user intends to and triggers it to trip. At all other times, it should continue its power supply to the line from the source. This is of huge importance especially during times of natural calamities, because during those calamities, huge environmental impact loads will be evident on the circuit breaker and critical electronic and electrical devices (for example, emergency fire fighting water sprayers) should function properly in order to minimize life and property loss [1]. In this research paper, a methodology involving mathematical modelling of the circuit breaker along with environmental impact forces, which will help to predict whether its latches would trip or not, under the specified conditions, is reported. If the results are positive (no nuisance tripping) with the necessary factor of safety, the product is good to go on with manufacturing trial runs and if the results are negative (that it trips), then corresponding design alterations should be made by an in-depth analysis of the mathematical results and the revised model is diagnosed again to see that the results are turning up to be positive. This will bring down the cost of practical testing, because the design engineer can now be sure from the mathematical 
results that the latch will not trip unnecessarily due to environmental impact forces and upon further practical impact testing, the result will always be a good to go. i.e., there will not be any scrap or rework form the impact testing department. So, the number of components of the lot to be tested can be significantly reduced leading to a reduction in the number of testing personnel and a reduction in the procurement of testing equipment and accessories. This, in turn will prove further more productive to the company because, time and space are always money incurring and by the concepts of lean manufacturing, these money incurring nonvalue added activities should not be carried upon. Also, the lead time of the switchgear can be significantly reduced. Through this research, investments in the testing department can be reduced and the space and time involved in testing of products can also be cut-down. This nuisance tripping can be prominent in places in which the circuit breaker is mounted near a high load press, in a sailing ship prominent to high pitching and yawing forces, during natural calamities, etc.

\section{Problem Description and Design Considerations}

Figure 1 clearly depicts the latch system taken for consideration. The latching system consists of two small mild steel (density $=7850 \mathrm{~kg} / \mathrm{m}^{3}$ ) cantilever beams hinged in a position as shown in Figure 1. Since the latches are kept at right angles, springs are to be used so that they keep pressing against each other at normal times.
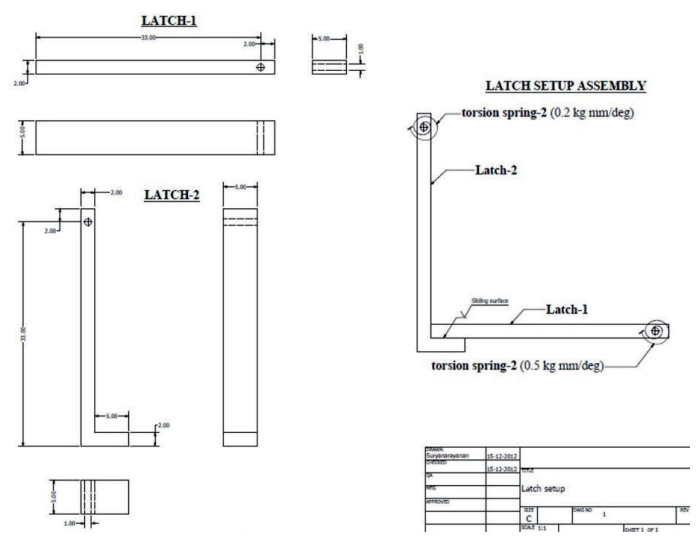

Fig. 1: Latch setup.

Here, either helical springs or torsion springs are used in the practical scenario. But, for our mathematical consideration, torsion springs are used because they have only one degree of freedom, i.e. their respective angular displacements. But, if the helical springs are considered to be used here, they will have two degrees of freedom, i.e. the linear displacement and the buckling tilt induced because of the angular displacements of the latches. Torsion springs are used in order to eliminate the complexity induced by the buckling tilt of helical springs. The torsion springs are assumed to have stiffness of $0.5 \mathrm{~kg} \mathrm{~mm} / \mathrm{deg}$ (or) $281.035 \mathrm{~N} \mathrm{~mm} / \mathrm{rad}$ for the one fixed to latch-1 and $0.2 \mathrm{~kg} \mathrm{~mm} / \mathrm{deg}$ (or) $112.414 \mathrm{~N} \mathrm{~mm} / \mathrm{rad}$ for the one attached with latch-2.

Since the nuisance tripping of the latches is described by the angular displacement of the latches above a permissible value and in a direction against the force applied by the torsion springs, it may be eliminated by using springs of high stiffness value. But, using springs of high stiffness would create problems during manual or intended tripping [2]. Because, in manual or intended tripping, the tripping force is given through some mechanical arrangement or through a bimetallic strip that makes the latches to displace against the springs so that the circuit is tripped. This applied force for manual tripping should be as low as possible in order to provide ease to the user. So, a small value of stiffness is considered to the springs. During intended or manual tripping, the latch-2 is made to displace against the spring (in CW direction) beyond a limit so that the latch-1 which is pressing against it will go down, causing tripping. This limit is based on the dimensions of the latching system. The permissible angular displacement calculation of latch-2 in order that tripping doesn't occur is discussed in section 2.1.

\subsection{Maximum permissible displacement}

The maximum permissible displacement for latch-2 is calculated through the trigonometric data shown in Figure 2. It is sufficient to check latch-2 alone for its displacement because, if the displacement of latch-2 exceeds the permissible value, it is understood that latch-1 will consequently exceed the permissible value of displacement because of its orientation and gets tripped because of its orientation. Also, the case in which latch-1 alone will be exceeding the permissible value of displacement, while latch-2 being in under 
safe numbers, is ruled out because of the configuration being reported here, i.e., latch-2 is holding up latch-1, which cannot displace without latch-2 displacing. The values of $\theta$ large and $\theta$ small are found to be 10.9540 and 1.84760 . On subtracting these two, the value of maximum permissible displacement (or) Olimit was found to be 9.1060 (or) 0.159 rad. So, if latch-2 doesn't displace beyond 9.1 degrees (or) $0.1495 \mathrm{rad}$, the latches will not trip unnecessarily. So latch-2 has to be checked whether it displaces more than 0.1495 rad or not, being applied with the considered force.

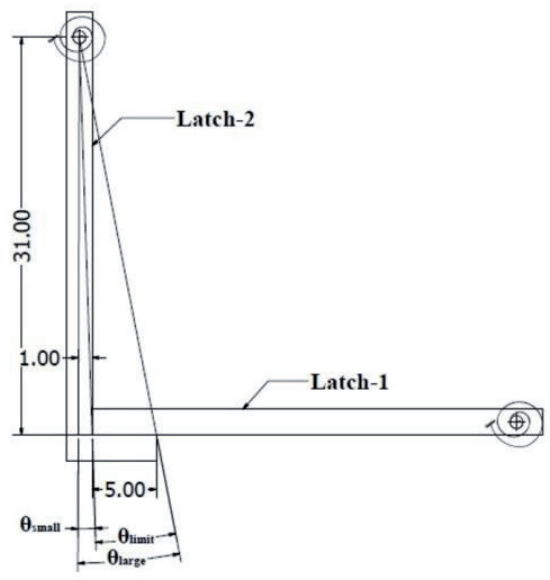

Fig. 2: Trigonometric data used to calculate maximum permissible displacement for latch-2.

\section{Modelling of Applied Force}

According to the IEC (International Electrotechnical Commission), some standards have been established to test circuit breaker latches [3]. One among them that caters to circuit breaker commonly used in house hold is chosen. It is given as follows:

- The force applied should be impulsive such that an immediate acceleration of $30 \mathrm{~g}$ (30 times the value of acceleration due to gravity) is imparted on the system.

- The force should be applied to the system for only 18 milli seconds.

Keeping in mind of these two data, the forces are modelled as follows:

- The mass of the latches are found out using their dimensions and density. Mass of latch-1, $m_{1}$ was found to be $0.002624 \mathrm{~kg}$ while mass of latch-2, $m_{2}$ was found to be $0.003016 \mathrm{~kg}$.
The found mass, when multiplied by the required acceleration of 30 times the acceleration due to gravity, will give the magnitude force in Newtons. By this, the magnitude of forces in acting in latch 1 and latch 2 are found to be $0.77224 \mathrm{~N}$ and $0.8876 \mathrm{~N}$. - The numerical value (magnitude) of forces to be applied has to be converted into impulsive forces. It is done by a method shown below.

According to literature survey, impulsive forces can be modelled in two ways. One is the Gaussian curve type and the other is the exponential decay type.

1) Gaussian curve:

$$
\mathbf{F}_{\mathrm{i}}=\mathbf{A}_{\mathbf{i}} \cdot \mathbf{e}^{\left\{\frac{-\left(t-\frac{\tau}{2}\right)^{2}}{(0.2 \tau)^{2}}\right\}}
$$

2) Exponential decay curve:

$\mathbf{F}_{\mathbf{i}}=\mathbf{A}_{\mathbf{i}} \cdot \mathbf{e}^{(-h . t)}$

where: $F_{i}$ is the equation of the force to be applied in the corresponding latch, $\mathrm{A}_{\mathrm{i}}$ is the magnitude of the force applied $(N)$, $\tau$ is the time interval to which the force is applied (seconds), $\mathrm{t}$ is the time instance and $\mathrm{h}$ is a constant corresponding to the required time interval. In order to choose one between these two, the compatibility of these two equations with respect to the different techniques in math. While analysing the former type, the Gaussian curve, it is found that $\mathrm{e}^{\text {t2 }}$ term is a problem. It can neither be used in Laplace transforms, nor can be used in the place to find the particular integral in the usual method of solving ordinary differential equations (ODE). Also, while explaining impulsive functions (or) dirac-delta functions, authors of some advanced engineering mathematics books have reported that those functions don't practically exist as rectangular pulses of very short intervals, but as an exponentially decaying function that decays approximately to zero in a very short interval of time $[4,5]$. So, the latter one, the exponentially decaying curve is chosen to model the force term in our case. The final modelling after choosing the type of curve is explained here.

So, it was decided to use a function of the format $\left[\mathrm{Ai} \times \mathrm{e}^{(-\mathrm{h} . t)}\right]$. Now, the value of ' $h$ ' corresponding to the time interval, i.e. 0.018 seconds is to be found. The use of the software 'MATLAB 2012' is mandatory here. There, a program to plot a function and see the graphs visually was created. It was then used to plot the equations, i.e. values of the equations, having 
random values of ' $h$ ' are plotted at various instances of time. While doing this, the value of ' $h$ ' was found using trial and error method to be 322 , in correspondence with our time interval of 18 milli seconds, i.e. when substituting the value of ' $h$ ' with the numeric 322 , the function was found to be decaying approximately to zero in approximately 18 milli seconds. The graph obtained in Matlab is shown in Figure 3.

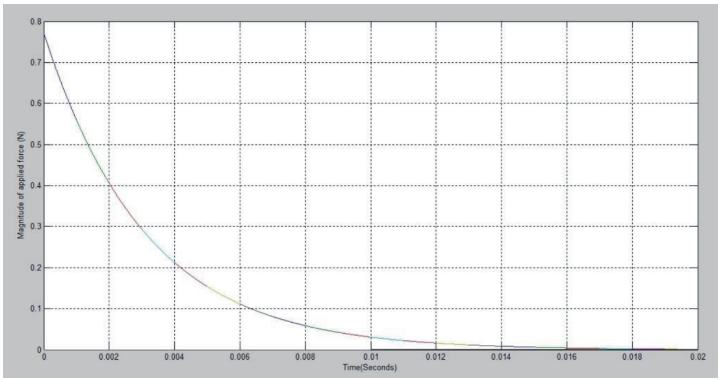

Fig. 3: Applied force (Latch-1 considered).

\section{Equation of Motion}

Consideration of an established equation of motion is very important, for all further calculations will be based on this. Basically, there are some ways by which the equations of motion can be modelled for complex spring-mass systems. They are Lagrange's equation of motion, Rayleigh's equation of motion and through construction of free diagrams. These methods, invented by the renowned mathematicians, Lagrange and Rayleigh are widely accepted methods to frame the equations of complex spring-mass systems that have inter-dependable multiple degrees of freedoms. In order to choose one among these two methods, careful interpretation of them is necessary. A textbook titled Schaum's outline on Mechanical Vibrations was very useful in understanding the types clearly [6]. Though the main outcome of the two will be similar, some differences are there in both, making them quite unique. Analysing from a point of view adhering to our requirements, the former one, i.e. the Lagrange's equation of motion was chosen because, the latter ones involves drawing free body diagrams in which there is a requirement that the different masses of the system has to be connected by a spring or a damper and that the point of connection of the spring/damper with the mass is to be rigid and immovable with respect to the masses. But in this case, there are latches that slide with respect to each other and it is not possible to model the connection between those two latches using any spring or damper with a rigid and immovable joint with the masses. The free body diagram method is also rejected due to our system experiencing physical sliding. So, the latter methods are rejected and Lagrange's method is chosen which conforms to all our requirements [7]. The Lagrange's equation of motion is represented as follows:

$$
\begin{aligned}
& \frac{\mathbf{d}}{\mathbf{d t}}\left(\frac{\partial(\text { K.E })}{\partial \dot{\theta}_{\mathrm{i}}}\right)-\frac{\partial(\text { K.E })}{\partial \theta_{\mathrm{i}}}+\frac{\partial(\text { P.E })}{\partial \theta_{\mathrm{i}}}+ \\
& +\frac{\partial(\mathrm{D} . \mathrm{E})}{\partial \ddot{\theta}_{\mathrm{i}}}=\mathrm{F}_{\mathrm{i}}
\end{aligned}
$$

where, K.E - Kinetic energy, P.E - Potential energy, D.E - Dissipating energy, $\theta_{i}$ - Angular displacement, $\dot{\theta}_{i}-$ Angular velocity, $\ddot{\theta}_{i}-$ Angular acceleration and $\mathrm{F}_{\mathrm{i}}$ - Force to be applied in the $\mathrm{i}^{\text {th }}$ member of the system.

\subsection{Modelling of Damper in the System}

The above equation (3) is based on the law of conservation of forces utilizing the kinetic, potential and dissipating energies of the different components of the system. A new term arises here called the dissipating energy. It corresponds to the damping property of the system. Even though it is not very visibly evident in the system, this has to be considered. Also, in our case, it happens to be that the damping elements are not of the usual shock absorbing type. Hence, a need arises here to find the damping coefficient in an unusual way [8]. It was hence referred that for systems which doesn't involves any visible or naked dampers, damping is supposed to happen due to the friction between the two surfaces in contact and that, the medium between them is also influential, even though very small in thickness [9].

\section{Evaluations and Calculations}

The Lagrangian equation of motion as given by the expression (3) is

$$
\begin{aligned}
& \frac{\mathbf{d}}{\mathbf{d t}}\left(\frac{\partial(\text { K.E })}{\partial \dot{\theta}_{i}}\right)-\frac{\partial(\text { K.E })}{\partial \theta_{i}}+\frac{\partial(\text { P.E })}{\partial \theta_{i}}+ \\
& +\frac{\partial(\text { D.E })}{\partial \ddot{\theta}_{i}}=F_{i}
\end{aligned}
$$

In the above equation, all unknown terms were evaluated using known data. Consequently, the 
evaluated expressions were applied with the corresponding differentiation or partial differentiation as given in equation (3). The various terms that were deduced are summarized in Table.1 and upon being applied with the corresponding differentiation, the deduced expressions to be substituted in equation (3) are summarized in Table. 2.

*Abbreviations are as given in section 5.1. The numbers in subscripts in the abbreviations denotes the latch numbers.

Table 1: Evaluation of basic terms

\begin{tabular}{ll|l|l|l|l|} 
Nomenclature* & K.E1 & K.E2 & P.E1 & P.E2 & D.E1, D.E2 \\
$\begin{array}{l}\text { Evaluated } \\
\text { expression }\end{array}$ & $1.428768 \times \dot{\theta}_{1}^{2}$ & $1.642212 \times \dot{\theta}_{2}^{2}$ & $140.5175 \times \theta_{1}^{2}$ & $56.207 \times \theta_{2}^{2}$ & $4.9575 \times\left(\dot{\theta}_{2}-\dot{\theta}_{1}\right)^{2}$ \\
\hline
\end{tabular}

Table 2: Differentiation of basic terms in accordance to the equation of motion

\begin{tabular}{|l|l|l|l|}
\hline Term in the equation of motion & \multicolumn{2}{l|}{ Evaluated expression } \\
\hline$\frac{\mathbf{d}}{\mathbf{d t}}\left(\frac{\partial(\text { K.E })}{\partial \dot{\theta}_{1}}\right)$ & $2.587536 \times \ddot{\theta}_{1}$ & $\frac{\partial(\text { P.E })}{\partial \theta_{2}}$ & $112.414 \times \theta_{2}$ \\
\hline$\frac{\partial(\text { K.E })}{\partial \theta_{1}}$ & $1.428768 \times \ddot{\theta}_{1}$ & $\frac{\partial(\text { D.E })}{\partial \dot{\theta}_{1}}$ & $9.915 \times\left(\dot{\theta}_{1}-\dot{\theta}_{2}\right)$ \\
\hline$\frac{\mathbf{d}}{\mathbf{d t}\left(\frac{\partial(\text { K.E })}{\partial \dot{\theta}_{2}}\right)}$ & $3.284424 \times \ddot{\theta}_{2}$ & $\frac{\partial(\text { D.E })}{\partial \dot{\theta}_{2}}$ & $9.915 \times\left(\dot{\theta}_{2}-\dot{\theta}_{1}\right)$ \\
\hline$\frac{\partial(\text { K.E })}{\partial \theta_{2}}$ & $1.642212 \times \ddot{\theta}_{2}$ & $F_{1}$ & $0.77224 \times \mathrm{e}^{(-332 . t)}$ \\
\hline$\frac{\partial(\text { P.E })}{\partial \theta_{1}}$ & $281.035 \times \theta$ & $F_{2}$ & $0.8876 \times \mathrm{e}^{(-332 . t)}$ \\
\hline
\end{tabular}

After substituting all found values and simplifying in the Lagrange equation (equation (3)), it gets transformed as

$$
\begin{aligned}
& \left(1.428768 . \ddot{\theta}_{1}\right)+\left(9.915 . \dot{\theta}_{1}\right)+\left(281.035 . \theta_{1}\right)-\left(9.915 . \ddot{\theta}_{2}\right)=0.77224 . \mathrm{e}^{(-322 . t)} \\
& \left(1.642112 . \ddot{\theta}_{2}\right)+\left(9.915 . \dot{\theta}_{2}\right)+\left(112.414 . \theta_{2}\right)-\left(9.915 . \ddot{\theta}_{1}\right)=0.8876 . \mathrm{e}^{(-322 . t)}
\end{aligned}
$$

\section{Solving the System of Equation}

The equation that is to be solved is a second order, simultaneous, non-homogenous ordinary differential equation. It cannot be solved manually in this same state. At least, it has to be transformed into a non-simultaneous state. This is done in order to make the set of equations liable to apply any mathematical procedures or techniques. This complexity arose due to the fact that the dissipating energy term involves the relative velocity term and this presents the relationship between the two latches in terms of frictional or dissipating energy. In order to make the system of equations (14) and (15) into a non-simultaneous one, a technique is adopted in which common terms of $\theta 1$ and $\theta 2$ are combined together in each expression and each combined multiple of both terms in an expression is multiplied with the multiple of the other term in the other expression, and the system of equation is added or subtracted such that any one of the term having equal or opposite multiples in both the expressions will get cancelled. After this procedure is carried upon, the system of equations will get transformed into a single equation, but with a higher degree. In this case, the system of equation given by equations (14) and (15) is cross-multiplied and added up such that the terms of $\theta 1$ will get cancelled. $\theta 1$ terms were made to get cancelled because it is evident from Figure 1 and section 3.1 that it is sufficient to check latch-2 alone for it displacement in order to determine the tripping of the latching system. The system of equation turns 
up to be equation (16) after being carried on with

$2.34634 \frac{\mathrm{d}^{4} \theta_{2}}{\mathrm{dt}^{4}}+30.44873 \frac{\mathrm{d}^{3} \theta_{2}}{\mathrm{dt}^{3}}+622.1325 \frac{\mathrm{d}^{2} \theta_{2}}{\mathrm{dt}^{2}}+3901.0468 \frac{\mathrm{d} \theta_{2}}{\mathrm{dt}}+31952.2685=126439.1472 . \mathrm{e}^{(-332 . \mathrm{t})}(16)$

Thus, the system of equations has become a single, non-simultaneous, non-homogenous, fourth order ordinary differential equation. It can be solved manually with four initial conditions. They are: At time $\mathrm{t}=0, \theta_{2}=\frac{\mathrm{d} \theta_{2}}{\mathrm{dt}}=\frac{\mathrm{d}^{2} \theta_{2}}{\mathrm{~d}^{2}}=\frac{\mathrm{d}^{3} \theta_{2}}{\mathrm{dt}^{3}}=0$.

A Matlab program was developed to find the roots the procedure stated here.

of the homogeneous equation based on which the complementary equation was developed and a program was developed to simultaneously solve the system of four equations which arises upon substitution of the initial conditions. Finally, the solution was found to be:

$$
\begin{aligned}
& \theta_{2}=\mathrm{e}^{(-1.43435 . t)} \cdot[(-0.1298 \cdot \cos (11.5767 . \mathrm{t}))-(0.07809 \cdot \sin (11.5767 . \mathrm{t}))]+ \\
& +\mathrm{e}^{(-5.0542 . t)} \cdot[(0.1298 \cdot \cos (8.63307 . \mathrm{t}))+(0.15934 \cdot \sin (8.63307 . \mathrm{t}))]+
\end{aligned}
$$

$+5.20953 .10-6 . \mathrm{e}^{(-322 . t)}$

Then, the result (equation (17)) has to be plot as a function of angular displacement of latch- 2 in radians with respect to time and the absolute maximum value of the graph has to be found. For this, a Matlab coding, similar to the one used in section 4.1 is used [10]. The graph displayed by the Matlab software is shown below in Figure 4.

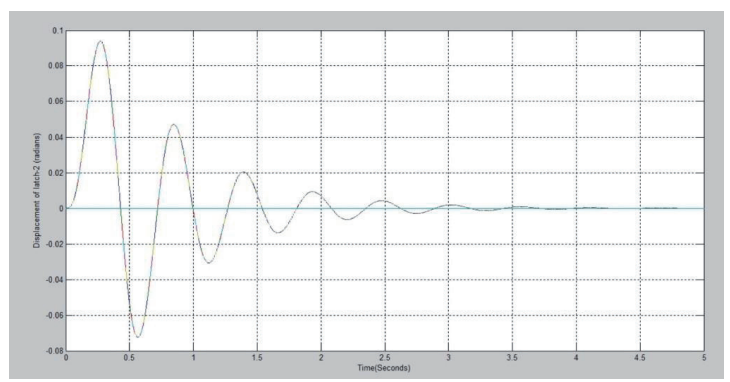

Fig. 4: Impulse response

Also, the absolute maximum and absolute minimum values of the graph were found to be 0.0939 radians and -0.0723 radians. The negative sign signifies that the displacement is in the opposite direction (ccw), to that which is kept as positive.

\section{Results and Discussions}

As found by the proposed mathematical model, the maximum angular displacement of the latch is $0.0939 \mathrm{rad}$ (or) $5.38^{\circ}$, which is well below than our maximum permissible displacement of $9.1^{\circ}$ (or) $0.1495 \mathrm{rad}$. So, the latch does not get tripped. Hence nuisance tripping does not occur in the latch system of given dimensions and material, when ap- plied with an impulsive acceleration of 30 times the acceleration due to gravity for 18 milli seconds. During this methodology, if the results turn up to be more than the permissible levels, it will mean that the latches will trip causing nuisance tripping to happen. In such a case, the resultant graph is to be carefully analyzed for its pattern and the time of tripping, and other such data can be found. Then, design alternatives are to be proposed by changing the dimensions such that the centre of gravity of the system further opposes the displacement caused by the impact forces, or by changing the material of the latches, changing the sub-components and so on. Then, the same procedure is to be followed and only when the results turn up to be positive (no nuisance tripping), the design is to be approved and proceeded for testing section and further to manufacturing section.

\section{Conclusion}

The methodology reported in this paper has successfully predicted whether a latching system in a circuit breaker trips unnecessarily or not, under the given circumstances. This methodology will prove very useful to many switchgear research and development centers and also to the researchers who are working in the vibrations and impacts field. One important thing to be said here is that researchers interested in this field, when intending to continue research in this area are asked to concentrate in developing codes in any software package in a better way so that the methodology becomes very user- 
friendly and time-saving. This is mainly because the engineers at any switchgear company will never find sufficient time to work in the mathematical part of solving the equation and that the mathematical solving ability of the engineers will also be a huge impact on the results. So, they will expect a userfriendly software package which will tell them the results of impact analysis by just giving the dimensions, material, orientation and shape and other such critical data as inputs. The mathematical software codes used in this paper are useful to an extent and further research should be carried on regarding this so that switchgear research and development gets to newer heights.

\section{Acknowledge}

The authors would like to thank Dr. K. A. Jagadeesh, Dept. of Mechanical Engineering and Ms. Nandhini, Dept. of Mathematics, PSG College of Technology, Mr. C. S. Kore, Mr. M. Kanakarajan and Mr. K. Senthil Kumar of SDDC, Larsen \& Toubro (Malumichampatty campus) for providing various resources to carry out the project.

\section{References}

[1] Meirovitch, L (1967). Analytical Methods in Vibrations, Macmillan Publishers. New York.

[2] Takács, G, Rohal'-Ilkiv, B (2012). Model Predictive Vibration Control. DOI: 10.1007/978-1-4471-2333-0_2, 2012, (C) Springer-Verlag London Limited.

[3] 60068-2-27 (2008). Basic environmental testing procedures - Part 2: Tests-Test Ea and guidance: Shock. International Electrotechnical Commission. (C) IEC:2008.

[4] Zill, D (2011). Advanced Engineering Mathematics. 4/e. Jones \& Bartlett Learning, MA. ISBN: 0763779660.

[5] Kreyzig, E (1992). Advanced Engineering Mathematics. 7/e. John Wiley \& Sons Inc., NY. ISBN: 0471553808.

[6] Kelly, SG (1996). Schaum's Outline of Mechanical Vibrations. McGraw Hill Professional, NY. ISBN: 0070340412.

[7] Yushkov, MP, Zegzhda, SA (2009). A New Method of Vibration Analysis of Elastic systems, Based on the Lagrange Equations of First Kind. TECHNISCHE MECHANIC. Band 18. Heft 2. 153-60.

[8] Xijun, L, Dajun, W, Yushu, C (1998). Approximate Analytical Solution of the Self-Excited Vibration of Piecewise-Smooth Systems Induced by Dry Friction. Acta Mechanica Sinca. ISSN 0567-7718.

[9] Rao, SS (1995). Mechanical Vibrations. 3/e. Addison-Wesley. Boston. ISBN: 0201526867.

[10] Young, P (2006). Solving Systems of Differential Equations.
Oct 24, 2006 [online], Available: http://amath.colorado.edu/ courses/2460/2008Spr/Worksheets/worksheet8.pdf 